\title{
Surfaces
}

\section{Squattage et terrains vagues}

\section{Marie Lessard}

Volume 6, 1996

LES ÉCONOMIES DISCURSIVES DU SAVOIR ET DE LA CULTURE DANS LE SILLAGE DE L'OEUVRE DE BILL READINGS

THE DISCURSIVE ECONOMIES OF KNOWLEDGE AND CULTURE, WITH CONSTANT REFERENCE TO THE WORK OF BILL READINGS

URI : https://id.erudit.org/iderudit/1064860ar

DOI : https://doi.org/10.7202/1064860ar

Aller au sommaire du numéro

\section{Éditeur(s)}

Les Presses de l’Université de Montréal

\section{ISSN}

1188-2492 (imprimé)

1200-5320 (numérique)

Découvrir la revue

\section{Citer cet article}

Lessard, M. (1996). Squattage et terrains vagues. Surfaces, 6. https://doi.org/10.7202/1064860ar
Résumé de l'article

La lecture des textes de Marguerite Duras, Kathy Acker et Irmtraud Morgner suggère la possibilité de penser le rapport entre pratiques d'écriture et autobiographie en termes de " pratiques de squattage ". Je veux expliciter ici ce qui m'apparait constituer l'intérêt d'un tel modèle lorsque l'on cherche à comprendre le rapport liant certaines pratiques d'écriture contemporaines à la tradition littéraire en général, ainsi que les modalités possibles d'une articulation politique entre pratiques culturelles et tradition, plus spécifiquement entre pratiques féministes et tradition.
Copyright @ Marie Lessard, 1996

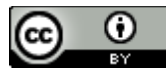

Ce document est protégé par la loi sur le droit d'auteur. L'utilisation des services d'Érudit (y compris la reproduction) est assujettie à sa politique d'utilisation que vous pouvez consulter en ligne.

https://apropos.erudit.org/fr/usagers/politique-dutilisation/ 


\title{
Squattage et terrains vagues
}

Marie Lessard

Université de Montréal

lessardm@ere.umontreal.ca

Surfaces Vol. VI. 215 (v.1.0F - 15/10/1996) - ISSN:

1188-2492

Tout texte reste la propriété de son auteur. Néanmoins, Surfaces demande d'être citée à l'occasion de toute autre publication du texte en question.

\section{RÉSUMÉ}

La lecture des textes de Marguerite Duras, Kathy Acker et Irmtraud Morgner suggère la possibilité de penser le rapport entre pratiques d'écriture et autobiographie en termes de pratiques de squattage . Je veux expliciter ici ce qui m'apparait constituer l'intérêt d'un tel modèle lorsque l'on cherche à comprendre le rapport liant certaines pratiques d'écriture contemporaines à la tradition littéraire en général, ainsi que les modalités possibles d'une articulation politique entre pratiques culturelles et tradition, plus spécifiquement entre pratiques féministes et tradition.

\author{
ABSTRACT \\ The reading of texts by Marguerite Duras, \\ Kathy Acker, and Irmtraud Morgner hints at \\ the possibility of thinking the relationship \\ between writing practices and autobiography \\ in terms of "squatting practices." In this text, I \\ seek to indicate what I consider to be the \\ interest of such a model in trying to \\ understand the link between certain \\ contemporary writing practices and the \\ literary tradition in general, as well as the
}


possible modalities of a political articulation between cultural practices and tradition, in particular when those practices derive from feminism.

Mon travail sur la question de l'autobiographie, telle que celle-ci se pose dans les textes de Marguerite Duras, de Kathy Acker et de Irmtraud Morgner[ $\underline{\mathbf{1}}$ ], m'aura menée à penser le rapport que l'on retrouve dans ces textes entre pratiques d'écriture et autobiographie en termes de pratiques de squattage . Je veux expliciter ici ce qui m'apparaît constituer l'intérêt d'un tel modèle lorsque l'on cherche à comprendre le rapport liant certaines pratiques d'écriture contemporaines à la tradition littéraire en général.

\section{Pratiques d'écriture et squattage de l'autobiographie}

Il me faut d'abord préciser comment je veux penser, ou plutôt repenser, ce terme d'autobiographie. En effet, si l'on se cantonne à l'acception traditionnelle du terme, on se trouve forcé, face à des textes tels ceux de Duras, Acker et Morgner, et plus généralement, au vu du phénomène littéraire contemporain, de se questionner sérieusement sur la pertinence qu'il y a, encore aujourd'hui, à parler d'autobiographie et d'entreprise autobiographique. Ainsi, pourquoi parler encore d'autobiographie, après les débats qui ont eu lieu autour de la notion de mort de l'auteur et de mort du sujet, ainsi qu'après la remise en question du régime réaliste de représentation qui permettait de maintenir en place la frontière entre vérité et fiction? Ou, pourquoi en parler encore, après qu'eut été démontrée par la critique féministe, la complicité de ce type d'entreprise avec une logique phallocentrique qui aura élevé au statut d'universel une conception particulière du sujet, la sienne, et opéré, de ce fait, l'exclusion de tout ce qui ne relevait pas de cette logique? Et que penser face à ces textes qui ne laissent intact ni le projet, ni les règles formelles, que nous associons généralement à l'autobiographie mais ne s'en réfèrent pas moins constamment à l'idée d'autobiographie? 
Or si, effectivement, ces textes indiquent qu'il existe bel et bien une possibilité de penser autrement

l'autobiographie et, pour être plus précis - car, c'est bien plutôt de cela dont il est question -, le rapport entre pratiques d'écriture et autobiographie, me suffirait-il simplement de chercher à réajuster, lectures théoriques et étude des textes à l'appui, une inadéquation, un décalage, qui se serait installé entre la réalité nouvelle des pratiques d'écriture autobiographiques contemporaines et une terminologie critique, des conceptions dépassées qui limiteraient notre manière de penser ce type d'écriture? Un tel travail, ayant comme horizon une simple adéquation entre langage critique et réalité littéraire, m'apparaît de peu de portée. Il ne s'agira donc pas ici de procéder à une revision générique féministe, qui permettrait, par exemple, une représentation plus juste de voix préalablement exclues ou l'établissement d'une tradition spécifiquement féminine. Il ne s'agira pas non plus de procéder à une revision générique postmoderne qui tiendrait compte de la sensibilité contemporaine et permettrait de mettre à jour, au diapason d'une sensibilité qu'on dirait postmoderne - - j'utilise ici ce terme dans un sens très vague qui lui est souvent attribué, équivalant plus ou moins au terme contemporain, ou encore, désignant ce qui vient après le moderne -, ou féministe, la conception traditionnelle de l'entreprise autobiographique et les règles formelles (génériques ou autres) qui en ont jusqu'ici déterminé la mise en écriture. Également, il m'apparaît dénué d'intérêt de déclarer l'autobiographie question dépassée - comme on dirait zone sinistrée -, ou encore de la déclarer réactionnaire, uniquement pour m'interroger ensuite sur une façon possible d'en réajuster le tir politique. Et d'ailleurs, à cet égard, une exigence s'impose, celle de reposer la question du politique afin d'échapper à une pensée qui ne nous laisserait comme alternative que de qualifier les pratiques d'écriture autobiographiques de politiquement réactionnaires ou de révolutionnaires. Pour l'instant, si intrigué ou interpellé par ces textes qui sont de plus en plus nombreux à revisiter la notion d'autobiographie, l'on cherche à penser une conception et une politique autres de l'autobiographie, en quels termes pourrait-on commencer à les penser? Et, à quoi pourrait ressembler, aujourd'hui, une pratique d'écriture autobiographique dont la politique pourrait être dite féministe?

La critique féministe des années 80 et 90 a soulevé la question d'une possible réappropriation de 
l'autobiographie aux fins d'un ordre du jour féministe. Elle a proposé deux alternatives. Tout d'abord, considérant les limites inhérentes à la conception de l'autobiographie dont nous avons hérité de la tradition, une première stratégie consisterait pour les femmes à envahir ce territoire jusqu'ici prioritairement masculin, c'est-à-dire à se gagner un droit d'entrée, de participation au jeu, à demander à être soumises aux mêmes règles que les hommes. En un sens, les femmes choisissent ici d'acheter des parts dans une société, de devenir actionnaires; elles se gagnent ainsi un droit de parole, c'est-à-dire un accès à l'écriture autobiographique et donc, également, à la possibilité de se produire elles-mêmes en tant que sujets, mais cela, uniquement dans le cadre des termes déjà prévus par la loi. On pourrait aussi dire qu'elles signent un contrat de location qui leur assure un droit à la jouissance des lieux, mais uniquement dans les termes d'un contrat qui définit quelles sont les limites étroites à l'intérieur desquelles elles peuvent intervenir sur ce territoire afin de le modifier. Bref, une plus grande ouverture est prévue afin de permettre l'inscription de la différence, mais les règles du jeu demeurent intactes: on réaménage un tant soit peu le territoire de l'autobiographie, on en adopte l'idée en la modifiant légèrement, mais ces réaménagements demeurent soumis aux lois préalablement dictées par le langage, l'usage, le genre. La différence se retrouve intégrée, assimilée dans l'autobiographie, mais sans que la définition de ce terme ne soit jamais remise en question.

Une seconde stratégie consisterait pour les femmes à se gagner l'accès à la propriété du territoire de l'autobiographie en signant un contrat qui établirait, non seulement leurs droits de propriété, mais également leurs droits à légiférer sur ce territoire. De cette manière, elles obtiendraient le droit de réaménager celui-ci comme elles l'entendent, selon leurs propres lois, c'est-à-dire de décider des règles qui prévaudraient en autobiographie : règles d'écriture, règles narratives, règles génériques nouvelles. Elles seraient libres d'instaurer un nouveau genre, une tradition alternative, qui trouverait son fondement dans une idée nouvelle de l'autobiographie et permettrait l'expression d'une expérience spécifiquement féminine. Mais l'adoption d'une telle stratégie ne va pas sans soulever quelques questions importantes. D'une part, si la souveraineté de tout propriétaire à légiférer sur son territoire est toujours limitée par les règlements de zonage municipaux, les lois provinciales, fédérales ou nationales, c'est-à-dire par des lois d'un ordre supérieur, on peut penser que, dans le cas de l'autobiographie, tout 
effort fait pour échapper à la tradition, pour tout effacer et repartir librement à zéro, demeurera vain puisque toute nouvelle tentative d'écriture demeurera soumise aux lois du langage. En un sens, le territoire de l'autobiographie demeure piégé, miné, par sa dépendance au langage.

Un autre problème concerne la loi qui aura cours sur ce territoire de l'autobiographie féminine. On pourrait se demander, par exemple, quelles seront les conditions d'admission aux frontières de ce nouveau territoire? Demandera-t-on que chacune présente une preuve de son appartenance au genre sexuel féminin? Ou bien le droit d'entrée sera-t-il accordé à toutes celles, et tous ceux, qui accepteront de se conformer à la loi du nouveau genre littéraire et souscriront à la nouvelle idée de l'autobiographie qui aura été mise de l'avant? On peut déjà prévoir qu'une difficulté similaire se présentera dans les deux cas, c'est-à-dire lorsqu'il s'agira de faire respecter la loi du genre littéraire ou celle du genre sexuel. D'une part, toute nouvelle loi risque fort de reproduire le même effet d'applatissement des différences qui caractérisait la loi de l'autobiographie traditionnelle, car, une fois encore, une différence singulière sera instaurée comme étant la différence qui compte, la seule à avoir droit de cité et d'accès à la représentation. D'autre part, si c'est la preuve d'appartenance au genre sexuel féminin qui est exigée de ceux et celles qui désirent avoir accès à ce territoire ce qui pourrait assurer qu'une fois ce critère respecté, des divergences d'opinion puissent être exprimées - on peut se demander comment une telle évidence devra être établie. Ainsi, lequel des trois je(s) identifiés par Lejeune[ 2 ] fera-t-il foi de l'appartenance au sexe féminin ou masculin? Ou encore, laquelle parmi les catégories identifiées par Judith Butler dans son livre Gender Trouble sera déclarée déterminante: anatomie sexuée, identité sexuelle ( gender identity ou gender performance ), c'est-à-dire trois dimensions contingentes - et possiblement dissonantes - d'une corporalité signifiante que la pratique du drag met en évidence[ $\underline{\mathbf{3}}$ ]. Quoiqu'il en soit, qu'une loi s'applique, celle de l'appartenance au genre sexuel ou celle de l'appartenance au genre littéraire, on devra se demander si ce n'est pas justement la loi du genre elle-même qui fait problème et, plus fondamentalement peut-être, la loi de la propriété - dans les deux sens de l'usage propre ou impropre d'une chose en regard d'une loi, ou encore, dans son sens plus classique de propriété légale -, qui fait véritablement problème. 
J'aimerais, à titre d'hypothèse, indiquer la possibilité de penser autrement le rapport entre pratiques d'écriture et autobiographie. Ce modèle m'est inspiré par ma compréhension de l'économie qui lie ces deux variables dans les textes de Kathy Acker. En effet, on ne peut éviter de s'interroger sur la nature particulière des rapports qu'entretiennent les pratiques d'écriture que l'on retrouve dans ces textes avec la tradition autobiographique, alors même que ceux-ci sont le produit d'une pratique d'écriture fondée sur le plagiat, le recyclage, l'imitation, la parodie et le pillage systématique des multiples sources textuelles offertes par la culture contemporaine et la tradition occidentale. Je fais l'hypothèse que le modèle que je développe ici permettrait également de rendre très bien compte du rapport qu'entretiennent avec l'autobiographie les pratiques d'écriture que l'on retrouve dans les textes de Duras et Morgner. Une alternative aux stratégies considérées ci-haut consisterait à décider d'occuper illégalement le territoire de l'autobiographie, c'est-à-dire de squatter [ $\underline{4}$ ] celui-ci. Ce verbe est défini comme suit: to settle on land, esp. public or unoccupied land without right or title.(...) to occupy illegally an empty abandoned, or condemned house, building, apartment, etc. [ $\underline{\mathbf{5}}$ ]. Le squatter, pour sa part, serait cette personne who takes unauthorized possession of unoccupied premisses [ $\underline{\mathbf{6}}$ ]. Qu'est-ce que ça pourrait bien signifier, pour une pratique d'écriture, que de squatter le terrain de l'autobiographie?

Dans un premier temps, une telle approche demande que l'on considère l'autobiographie - et peut-être même la tradition littéraire et la culture occidentales dans leur ensemble - comme un terrain laissé vacant et à l'abandon par un propriétaire absent ou, du moins, fort difficile à retracer. Dans le contexte actuel de mondialisation et de déclin de l'État nation, ce modèle ne me semble pas du tout inapproprié. En effet, on pourrait dire, figurativement, que l'Etat nation, qui s'est longtemps proclamé le propriétaire attitré de ces deux valeurs que sont la culture et la littérature, se réclame de moins en moins d'elles. Ayant perdu la fonction idéologique centrale qui fut la leur (ainsi, la production d'un sujet national et la production d'un sujet de droit individuel) et ne faisant plus, à la limite, aucune différence pour l'État nation[ 7 ], la culture et la littérature se retrouvent avec un statut qui n'est peutêtre pas si loin de celui du terrain vague[ $\underline{\mathbf{8}}$ ], tour à tour bidonville, immeuble abandonné et dépotoir de la culture occidentale. En effet, si personne ne réclame plus la propriété de ces terrains vagues, si n'importe qui peut 
les occuper, c'est bien parce que leur propriété n'est plus liée à aucun enjeu en regard d'une loi du marché qui n'est plus nationale mais mondiale. Et on peut penser que si leur occupation représentait encore un enjeu stratégique quelconque, un mécanisme de contrôle serait établi afin de limiter l'accès à ce territoire: aucun squatter ne serait toléré. Enfin, des investissements seraient faits afin de rendre possible une protection et un développement de ces territoires de la culture et de la littérature et afin de contrôler les modalités de leur aménagement. Mais, qui se soucierait d'investir afin de protéger un territoire qui ne porte plus à conséquence? On procédera plutôt, par exemple dans les universités, à d'importantes coupures budgétaires qui menaceront vite l'existence des départements d'études littéraires. Les dénominations de territoire abandonné ou plutôt de terrain vague de la culture prennent ici tout leur sens. En effet, on ne peut plus véritablement parler de territoire puisque, dans le cas qui nous intéresse, le site en question n'est plus revendiqué - c'est à dire également défendu et protégé - ni par une communauté humaine donnée qui s'identifierait à celui-ci[ $\underline{\mathbf{9}}$ ], ni par une collectivité politique nationale, ni par une autorité ou une juridiction donnée. Bref, on n'a plus affaire à un territoire du tout.

Pour retourner à la question plus spécifique de l'autobiographie, qu'est-ce que cela signifierait, pour une pratique d'écriture, de squatter ce terrain vague qu'est désormais l'autobiographie, comme l'on squate, à Paris ou à Hamburg[ 10 ], les immeubles abandonnés? Cela signifierait, par le biais de l'écriture, occuper illégalement ce site abandonné et le réaménager en pillant tout ce qu'on y trouve, une pratique exigeant du squatter la capacité de transformer ce qu'il rencontre, de le réutiliser à des fins immédiates qui seront fort probablement différentes de celles prévues à l'origine, des fins hétérogènes et métisses qui ne sont prévues ni par les codes des règlements municipaux, ni par les normes littéraires ou génériques. Dans le cas qui nous intéresse, ce modèle du squattage s'avère particulièrement intéressant puisqu'il évoque tout à la fois plusieurs termes, du pillage au recyclage, de l'anthropophagisme au phagocytage en passant par le parasitage, qui ont été proposés, tour à tour, par la critique comme autant de modèles qui pourraient nous aider à repenser les rapports possibles à une tradition littéraire européo-centrique, ou phallogocentrique.

Penser un tel rapport à l'autobiographie impliquerait en premier lieu que l'on envisage en aborder l'étude sans avoir recours à aucune idée de l'autobiographie[ $\underline{11}$ ]. 
D'une part, parce que les termes de cette occupation ne sont prévus par aucune loi. En effet, aucun contrat n'a pu être signé. De plus, si, en principe, ce site est toujours soumis à la loi, de facto, personne ne se soucie plus de savoir si celle-ci est respectée ou non: il sera donc impossible de penser la politique de cette occupation en termes de confrontation, de transgression, de subversion ou de révolution face à une quelconque loi, une idée, une politique de l'autobiographie qui aurait eu cours. D'autre part, comme le squatter ne peut acquérir de droit légal sur le site ou le bâtiment qu'il occupe, ni de droit de le gouverner et d'en rédiger les lois, il ne ne peut se permettre, non plus, d'ériger en loi sa propre idée de l'autobiographie.

J'insiste sur l'élément incontournable d'illégalité qui se trouve associé à cette pratique. Celle-ci, en effet, ne s'autorise pas de la loi. Bien sûr, comme on l'a pressenti plus haut, les menaces de se faire évincer par les forces de l'ordre sont faibles: personne ne prendra plus la peine de vous contester le recours à ce terme ou de vous interdire une utilisation transgressive de sa tradition. La loi tolèrera la présence du squatter tant et aussi longtemps que le terrain occupé, ici celui de l'autobiographie, demeurera sans valeur pour l'ordre symbolique et économique. Entre temps, ce dernier ne possède aucun titre de propriété, aucune forme de légitimation autre que sa pratique d'occupation. $\mathrm{Ne}$ possédant pas le droit de fixer, par une définition ou un ensemble de lois, ce qu'est l'autobiographie, le squatter ne pourra légitimer un appel à ce terme que par une référence aux pratiques d'écriture, singulières, ponctuelles et changeantes qui seront les siennes. Pour en revenir à mon idée de départ, squatter ce terrain vague qu'est désormais l'autobiographie ce serait penser l'autobiographie sans avoir recours à une conception, même nouvelle, de celle-ci: l'autobiographie n'est plus, à ce titre, que ce qu'en font - et parviennent à en faire - les textes. L'autobiographie n'aurait plus d'identité propre, elle ne serait plus définie que par des performances singulières et des pratiques locales.

De ce fait, le squatter n'aura-t-il donc de comptes à rendre à personne, ne sera-t-il redevable envers aucune loi? Il est important de rappeler ici tout ce qu'un tel mode d'occupation a de nécessairement précaire: c'est un mode d'occupation temporaire, sans statut, incertain, qui doit se passer d'autorisation et de légitimité. Il faut surtout souligner le fait qu'il ne renvoie jamais à une position de souveraineté ou d'autonomie, qu'il ne renvoie justement pas à un exercice de pure expérimentation ludique. Tout au contraire, il définit une position de forte 
hétéronomie: la forme que prendra cette occupation dépendra de ce qu'on voudra bien en faire, mais, également, de ce qu'on pourra en faire. Si j'ai tout d'abord parlé d'une pratique d'écriture qui squatte et hante activement le site de l'autobiographie, il faut voir que celle-ci se retrouve aussi hantée par l'autobiographie, c'est-à-dire par la tradition. Squatter le terrain vague de l'autobiographie, c'est envisager occuper et aménager un terrain à usage indéfini mais, en même temps, fortement déterminé. Car il faut compter avec la matérialité de ce terrain qui demeure marqué, miné, par ses occupations précédentes; un terrain sur lequel la temporalité s'inscrit comme une accumulation de traces. Ainsi, la tradition, avec tout son bagage de présupposés philosophiques, de prescriptions formelles et génériques, y aura laissé son empreinte. Si, au premier abord, il peut sembler que toute liberté est laissée au squatter pour inventer, transgresser ou réécrire la tradition, il faut se souvenir que c'est aussi à partir de ce qui aura été laissé derrière par ses prédécesseurs que tout nouveau texte s'écrira. Ainsi, les déterminations rencontrées en cours d'écriture, et avec lesquelles l'écriture devra ruser, seront multiples et imprévisibles. Il faudra compter avec les sentiers déjà tracés qui inviteront la simple répétition et, également, avec l'illusion de la possibilité d'une innovation absolue. Mais, du fait de sa position précaire, le squatter ne pourra éviter la confrontation avec certains impératifs tout à fait pragmatiques: s'il doit ruser avec la tradition, ce sera toujours en fonction d'une demande pour des solutions locales et ponctuelles (Kathy Acker parlera d'une production de myths to live by [ 12 ]). Selon moi, prendre sérieusement en considération cet ordre de détermination permettrait de réintroduire, dans notre pensée de l'autobiographie, une exigence à la fois pragmatique, ponctuelle et situationnelle, c'est-à-dire en ce sens, une exigence politique.

M'intéressant à l'éventail des rapports possibles entre pratiques d'écriture et autobiographie, je m'interroge plus particulièrement sur les rapports de négociation qui s'instaureront entre ces pratiques d'écriture et 1) l'autobiographie en tant qu'instance narrative visant l'inscription de l'identité, 2) l'autobiographie telle qu'elle se trouve nécessairement marquée par la question du genre et enfin, 3) l'autobiographie telle qu'elle se trouve nécessairement marquée par la question du gender ou de la différence sexuelle. En d'autres mots, je comprendrai la narrativité, le genre et le gender, comme autant de termes ayant jusqu'à présent servi à nommer, à cartographier ce lieu incertain qu'est l'autobiographie. Toute tentative d'occupation nouvelle de celui-ci, celle 
que vise ce travail de réflexion, comme celle des textes de Duras, Acker et Morgner, ne pourra faire l'économie d'une négociation avec ces trois termes.

Dans un premier temps, on pourrait se demander comment pourrait s'articuler, dans le cadre de ce rapport entre écriture et autobiographie en tant qu'instance narrative, une pensée autre du sujet et de l'identité. Car il existe bel et bien une politique de l'autobiographie traditionnelle qui détermine les règles de représentation, et donc de production de sujets et d'identités, et qui prête à ces règles toute l'évidence et la transparence de faits naturels. Or l'articulation entre pratiques d'écriture et autobiographie pourrait fonctionner comme le site privilégié d'une lutte politique touchant cette question. Je fais l'hypothèse que c'est, entre autres, un tel site politique qui se trouve squatté, occupé et réaménagé, par des pratiques d'écriture telles celles de Duras, Acker et Morgner. Même si les angles d'attaque et les stratégies utilisés s'avèrent différents d'une oeuvre à l'autre, c'est du côté d'une remise en question, d'une dénaturalisation généralisée de telles règles implicites - qui dans notre société déterminent bien plus que la production de récits littéraires -, que l'on peut minimalement situer la politique des textes de Duras, Acker et Morgner.

Dans un deuxième temps, il faut prêter attention au rapport au genre qui, dans chacune des oeuvres étudiées, n'est jamais absent, neutre, ou indifférent. On ne peut simplement pas éviter d'y réfléchir. Le texte de Jacques Derrida, La loi du genre [ 13 ], ouvre une voie intéressante, pour qui cherche à aborder autrement la notion de genre. Rapidement, alors même que celle-ci nous renvoie spontanément à des notions de limite, de norme et d'interdit, Derrida propose que nous nous intéressions plutôt, par un étrange retournement des choses, à l'ancrage de celui-ci dans ce qu'il nomme la loi de la loi du genre, c'est-à-dire dans un principe de contamination, une loi d'impureté, une économie du parasite (...) une sorte de participation sans appartenance [ 14 ], bref, dans cette impossibilité fondamentale qu'il y aurait à respecter tout interdit, toute frontière. Dès lors, au lieu de penser le genre comme un appareil dont la fonction principale serait d'ordonner, de maintenir à l'ordre, de contrôler les frontières, on le penserait à partir de cet inévitable et incessant débordement des frontières qui appelle et fonde tout travail ultérieur d'ordonnance des genres. L'une des façons de penser la résistance du genre aux diverses analyses qui s'efforcent d'en fixer la forme ou l'identité - ou encore, de penser la propension de 
l'autobiographie à demeurer terrain vague ou variable incertaine - consisterait à considérer celle-ci comme relevant de cette loi de la loi du genre qu'invoque Derrida. Or, ce principe d'incessant débordement des frontières, de contamination et d'impureté, cette économie du parasite - je parlais de squattage -, cette participation sans appartenance - je parlais d'occupation ponctuelle, temporaire et de l'impossibilité d'obtenir un droit de propriété -, renvoie exactement à ce qui m'intéresse tout particulièrement dans les textes de Duras, de Acker et de Morgner, c'est-à-dire à leur mode d'occupation de l'autobiographie en tant que genre. Un tel refus d'assigner l'autobiographie à demeure et de fixer son identité m'apparaît comme la seule façon possible de penser ensemble les pratiques d'écriture qui sont ici à l'étude et la notion d'autobiographie. Notons à cet égard que la problématique identité du genre à luimême, ou encore de celle du texte à lui-même - lorsqu'il y a, par exemple, répétition -, du texte au nom propre dont il porte la signature - lorsqu'il y a plagiat -, ou encore du sujet à lui-même - dans l'économie du texte autobiographique -, nous renvoie sans cesse à considérer l'intérêt qu'il y aurait à privilégier non plus les termes d'identité et de propriété mais plutôt ceux de pratiques et de performances.

Enfin, je souligne que lorsque je parle d'ordonnance des genres, j'entends ordonnance des genres littéraires bien sûr, mais également ordonnance des genres sexuels (ou genders), dont l'intersection avec l'écriture autobiographique $m$ 'intéresse tout particulièrement dans ma lecture des textes. Les critiques féministes ont clairement montré que l'autobiographie, qui a longtemps été présentée comme le discours d'un sujet universel et neutre, était en réalité une pratique d'inscription des récits qui, si elle pouvait être définie comme étant le propre de la tradition moderniste, pouvait également l'être comme étant le propre de la tradition phallologocentrique dénoncée entre autres par Derrida. Dans cette optique, il faudrait penser l'autobiographie comme une pratique d'inscription des récits qui se trouve soumise à la loi d'une détermination générique, non plus seulement littéraire mais également sexuelle. Mais, de quelle manière le genre sexuel marque-t-il effectivement ces rapports? Comme on l'a vu plus haut, la notion d' autobiographie féminine soulève quelques problèmes. Je fais l'hypothèse que le jeu de la différence sexuelle relèvera d'une réalité complexe dont l'opposition binaire classique, et exclusive, entre appartenance au genre féminin et au genre masculin ne suffira pas à rendre compte. Il faudrait distinguer entre, d'une part, l'existence de cette différence indéterminable 
que serait la différence sexuelle, et qui renverrait à une sorte de loi de la loi du genre, et de l'autre, l'ensemble de toutes ces lois et règles génériques qui tentent vainement d'ordonner et de maintenir à l'ordre, par le biais de lois d'appartenance et d'un contrôle des frontières, le jeu de cette différence. On pourrait ainsi penser le genre sexuel, à l'instar du genre littéraire, comme dépendant de cette loi de la loi du genre, qui serait la condition même de possibilité de toute tentative visant à réglementer et à contrôler cet insaisissable jeu de la différence.

Dès lors, au lieu d'aborder la question du genre à partir d'une quelconque loi du genre, je choisis de l'aborder à partir de cet inévitable et incessant débordement des frontières qui appelle et fonde tout travail ultérieur d'ordonnance des genres. Je fais l'hypothèse qu'il en serait de même de l'identité du texte à lui-même, du sujet à lui-même, du texte à son genre ainsi que du texte ou du sujet à un genre sexuel. Dans chaque cas, une identité se trouve fixée par la loi du genre, qui repose elle-même sur sa propre impossibilité. En effet, peut-on parler du propre et de l'appartenance, que ce soit à un nom, une signature, un je autobiographique, un genre littéraire ou un genre sexuel, s'il n'y a pas déjà de l'altérité, de l'impropre, de l'impureté à partir desquels pourrait se cristaller ce sentiment d'appartenance, se construire ce propre, dont l'impossibilité même s'avère la condition fondatrice de la constitution identitaire?

Il faudrait considérer en quoi cette suggestion de Derrida, de penser à partir de cet autre du genre, se distingue d'une pensée de l'autobiographie qui prendrait comme point de départ, à la suite de Paul de Man, l'opposition entre discours et figure. Dans cette optique, il faudrait penser l'autobiographie, la narration, le genre et le gender comme autant de terrains vagues qui seraient marqués non seulement par l'équivalent d'une loi du genre , mais également, hantés par une plus fondamentale loi de la loi du genre. Ainsi, il faudrait considérer que ceux-ci porteront, non seulement la trace des règles et des lois discursives imposées par la tradition, mais seront également hantés par cette inévitable figuralité du langage qui fonde la possibilité même d'une inscription matérielle de la tradition dans le langage. Anticipant sur ma considération de l'autobiographie en tant que figure, j'aimerais suggérer qu'il faudrait considérer de quelle manière la question de la performativité politique de l'autobiographie se trouverait reformulés si, suivant la suggestion faite par Paul de Man dans Autobiography as Defacement [ $\underline{\mathbf{1 5}}$ ], l'on décidait de considérer ce qui, dans l'autobiographie - 
et possiblement dans la narration - ne relève plus de l'ordre du discours, mais de l'ordre de la figure. Si l'on emprunte la définition de ce terme que nous suggère Bill Readings dans le glossaire de son Introducing Lyotard, le discours serait, "the condition of representation to consciousness by a rational order or structure of concepts."

Comprise comme appartenant à la condition du discours, et se trouvant donc limitée par celle-ci, l'autobiographie n'appréhendrait les choses qu'en termes de leur représentabilité par, ou dans, les termes de son propre système discursif, c'est-à-dire comme des significations que le discours serait apte à exprimer[ $\underline{\mathbf{1 6}}$ ]. Au contraire, comprise comme relevant de l'ordre de la figure, l'autobiographie appartiendrait à cet autre du discours qui, se trouvant toujours nécessairement présent dans celui-ci, travaille contre lui et déplace l'ordre de la représentation[ 17 ]. Elle pourrait, de cette façon, être comprise comme la trace persistente et irréconciliable d'un espace ou d'un temps qui serait radicalement incommensurable avec le temps et l'espace relevant de la signification discursive. La figure de l'autobiographie, ainsi pensée, se situerait à ce point où les oppositions par le biais desquelles le discours fonctionne sont ouvertes à cette radicale hétérogénéité ou singularité diversement évoquées dans les écrits de Lyotard par les termes d'événement, d'inconscient, d'anachronisme postmoderne ou de sublime[ $1 \mathbf{8}$ ]. La question de la valeur politique de l'autobiographie, posée comme figure de lecture ou figure d'écriture, s'en trouverait radicalement déplacée. Dans cette optique, la discursivité de l'autobiographie, de la narration, du genre, ou encore du gender, ne représenterait plus que la somme des traces laissées sur ces sites mouvants par de précédentes tentatives d'occupation, d'appropriation et de cartographie, sites qu'elles ne seraient, par ailleurs, jamais parvenues à fixer ou à contrôler. Dès lors, ne pourrait-on pas considérer ces instances, et également celles du nom propre, du texte et de la signature, comme autant de terrains vagues devenus d'autant plus ouverts à des pratiques de squattage multiples qu'ils ne sont plus revendiqués ou défendus par quelque instance institutionelle, étatique ou juridique que ce soit qui ait les moyens de les défendre?

De façon concrète, si l'on refuse de comprendre le gender, ou bien comme un simple ensemble de conventions formelles qui seraient modifiables à l'infini - par le biais de la culture -, ou bien comme une essence ou une forme organique inchangeable - déterminée par la nature -, que nous reste-t-il comme option? On doit 
s'interroger sur les possibilités ouvertes par une sorte de pratique de participation sans appartenance [ 19] ou, selon le modèle proposé plus haut, sur une sorte de pratique de squattage de ces différentes instances. Une fois encore, squatter ces divers terrains vagues ce serait occuper illégalement et de façon temporaire des sites à la fois incertains et minés, c'est-à-dire déjà marqués par des occupations précédentes, les occuper par le biais de pratiques - performances textuelles ou pratiques d'écriture - temporaires et ponctuelles, et ce sans avoir recours à une idée ou à une définition. De telles pratiques ne renvoient pas à une position de souveraineté, d'autonomie ou à un exercice de pure expérimentation ludique - ainsi, il existe bien quelque chose de l'ordre de la différence sexuelle ainsi qu'une tradition philosophique qui nous dicte notre compréhension de celle-ci -, mais à une position d'hétéronomie[ 20 ]. La forme que prendront ces diverses pratiques de squattage dépendra, non seulement de ce qu'on voudra bien en faire mais également de ce qu'on pourra en faire, c'est-à-dire, entre autres, de ce qu'on trouvera de déjà inscrit sur ces sites et de la façon dont on interprétera ceux-ci à la lumière des impératifs politiques pragmatiques et situationnels qui s'imposeront au travail d'écriture ou de pratique culturelle. Ainsi, en ce qui concerne le gender, la seule identité qui naîtra de l'occupation de ces sites incertains et mouvants sera définie par les pratiques d'occupation qui, seules, seront à même d'ouvrir ainsi des possibilités nouvelles pour une subjectivité qui se trouverait diversement marquée par le genre.[ 21 ]

\section{Post-scriptum}

Ce texte rend compte, en partie, du travail de rédaction fait à l'été 1995 en vue de la préparation de mes examens de doctorat à l'université de Montréal. J'aurais dû passer ces examens un an plus tôt, soit, plus exactement, en novembre 1994. La disparition de Bill Readings vint bouleverser ces projets et en remettre en cause le sens même. Comment persévérer, en son absence, dans cette aventure de la pensée dont il m'avait redonné le goût? On pense d'abord que continuer, c'est un peu oublier, c'est un peu trahir. Et puis, l'on pense que refuser d'avancer, c'est aussi trahir. Car refuser d'assumer la responsabilité pour le projet entrepris, ce serait faire comme si ce cheminement, cet 
accompagnement d'une qualité et d'une complicité si rares dont j'avais profité, n'avait pas eu lieu.

Bien avant qu'il n'y ait eu à nouveau des raisons pour continuer, il y aura eu cette évidence qu'il le fallait, tout simplement, et, aussi, le sentiment d'une dette impossible à payer. Ce n'est qu'en retournant à l'écriture, ainsi en rédigeant la première version de ce texte, que s'imposa finalement l'évidence d'un accompagnement, d'une présence, qui se poursuivait malgré l'absence. Aujourd'hui, de Berlin, où je ne serais pas venue, de Berlin où il aurait dû être lui-même cette année, je réalise à quel point mon travail ne pourra se poursuivre qu'à partir de l'héritage laissé en moi par le dialogue constant, actuel ou virtuel, qui m'attacha à la pensée de Bill Readings durant ces quatre années qu'il passa parmi nous à Montréal.

\section{NOTES}

1. Irmtraud Morgner, Allemagne de l'Est (1933-1990), Kathy Acker, U.K. et U.S.A., (1948-...) et Marguerite Duras, France (1914-1996).

․․ Lejeune, Philippe, Le Pacte autobiographique , Poétique, 14, 1973.

3. Judith Butler Gender Trouble. Feminism and the Subversion of Identity. Routledge, New York, 1990, p. 137.

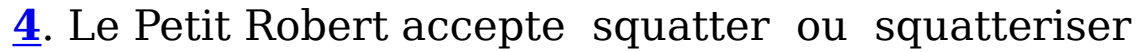
comme forme francaise du verbe anglais to squat. On parlera également d'un squatter en faisant référence à la personne qui s'adonne à une telle activité. On dira également, faire du squattage .

5. Webster's New World Dictionary of American English. Webster New World, Cleveland \& New York, 1988 (Simon \& Schuster Inc.), p. 1301.

6. The Concise Oxford Dictionary. Clavendon Press, Oxford, Oxford U. Press, 1990, p. 1182.

7. Consulter à cet égard l'intéressante analyse de Bill Readings dans The University in Ruins, Harvard University Press, 1996. 
8. Leur valeur symbolique potentielle est toujours bien réelle, mais elle est désormais comparable à celle de n'importe quel bien économique.

9. Cette forme de revendication demeure tout de même plus réelle en Europe de l'Ouest que sur le continent nord-américain où une telle identification ne suffit pas à faire obstace à l'abandon des politiques d' approvisionnement de ces territoires par les politiques étatiques. Par ailleurs, les efforts de revendication de la culture par des pratiques individuelles (plutôt qu'étatiques ou juridiques) telles celles qui ont été traditionnellement encouragées par les départements de littérature nationale demeurent assujettis à une conception fétichisée de la culture dont on cherche surtout à s'approprier le capital symbolique. On peut prévoir que ces efforts, en autant qu'ils demeureront le fait d'individus isolés et resteront prisonniers d'une logique et d'un horizon d'appropriation et de conservation - on vise à devenir dépositaire d'une origine, la culture ou la tradition - s'avèreront également insuffisants pour empêcher la généralisation de cette sorte de devenir terrain vague de la culture dont il est ici question. Mon argument prend pour point de départ qu'un tel phénomène est inévitable et qu'il s'agit surtout de trouver des stratégies permettant, face à une telle situation, de penser les modalités politiques possibles d'un rapport à la tradition.

10. Hamburg: Gerichtsentscheid über den Pachtvertrag für die Hafenstrasse: Der Anfang vom Ende eines Symbols. Zehn Jahre nach der Häuserbesetzung in St. Pauli scheint aus dem einstigen politischen Streitobjeckt nurmehr ein juristisches Problem geworden zu sein , Süddeutsche Zeitung, Nr. 255, Seite 3, Dienstag, 5 November 1991.

11. Bill Readings, "Identity Crisis: The University and Culture", ACCUTE Newsletter,1993.

12. Devoured by Myths. An Interview with Sylvère Lotringer in Kathy Acker, Hannibal Lecter, My Father. ed. Sylvère Lotringer, Semiotext(e) Native Agents Series, New York, 1991.

13. Conférence présentée au Colloque International sur le genre à l'Université de Strasbourg en juillet 1979. Publiée dans Glyph. Textual Studies. Actes du Strasbourg Colloquium: Genre. A Selection of Papers. The Johns Hopkins University Press, Baltimore and London, numéro 7, 1980. 
14. (...) perturbation essentielle qui peut être qualifiée d'impureté, corruption, contamination, décomposition, perversion, déformation, cancérisation même, prolifération généreuse ou dégénérescence . Dans Jacques Derrida, La loi du genre, Glyph. Textual Studies. Actes du Strasbourg Colloquium: Genre, p. 179-80.

15. De Man, Paul, Autobiography as Defacement, The Rhetoric of Romanticism. Columbia University Press, New York, 1984. (Reprise de: Paul de Man, Autobiography as De-facement. Modern Language Notes 94, 1979, 919-30).

16. Bill Readings, Introducing Lyotard. Art and Politics. Routledge, New York, 1991, p. xxxi et p. 63.

17. Ibid., p.xxxi.

18. Ibid., p. xxxi.

19. Derrida, La loi du genre, op..cit., p. 194.

20. Voir Judith Butler, Bodies that Matter: On the Discursive Limits of Sex, Routledge, New York, 1993.

21. Voir Jacques Derrida et Christie McDonald, Choreographies, Jacques Derrida and Christie V. McDonald , dans Diacritics, vol.12, The John Hopkins University Press, 1982, pp. 66-76: (...) I would like to believe in the multiplicity of sexually marked voices. I would like to believe in the masses, this indeterminable number of blended voices, this mobile of non-identified sexual marks whose choreography can carry, divide, multiply the body of each individual, whether he be classified as man or as woman according to the criteria of usage. (...) In a quite rigorous sense, the exchange alone could not suffice either, however, because the desire to escape the combinatory itself, to invent incalculable choreographies, would remain.

Accueil Surfaces | Table des matières | Recherche Surfaces Home Page | Table of Contents | Search

PUM | Livres | Revues | Publications électroniques | Vente et distribution 